\title{
How to measure energy of LEReC electron beam with magnetic spectrometer
}

\author{
S. Seletskiy
}

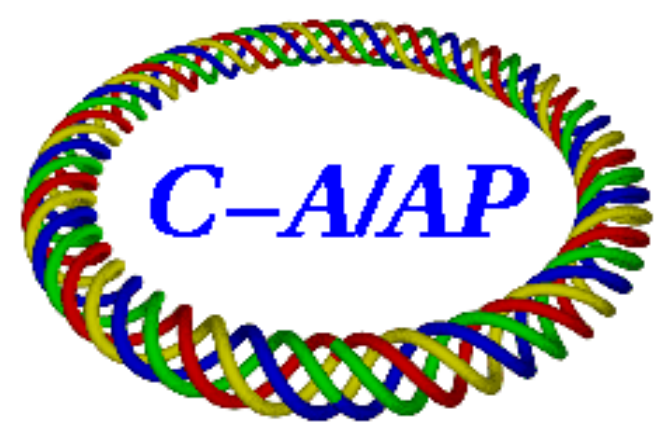

\section{Collider-Accelerator Department Brookhaven National Laboratory Upton, NY 11973}

\author{
U.S. Department of Energy \\ Office of Science, Office of Nuclear Physics
}

Notice: This document has been authorized by employees of Brookhaven Science Associates, LLC under Contract No. DE-SC0012704 with the U.S. Department of Energy. The United States Government retains a nonexclusive, paid-up, irrevocable, world-wide license to publish or reproduce the published form of this document, or allow others to do so, for United States Government purposes. 


\title{
How to measure energy of LEReC electron beam with magnetic spectrometer
}

\author{
April 11, 2016 \\ S. Seletskiy
}

\section{Introduction}

For successful cooling the energies of RHIC ion beam and LEReC electron beam must be matched with $10^{-4}$ accuracy. While the energy of ions will be known with required accuracy, ebeam energy can have as large initial offset as $5 \%$.

The final setting of e-beam energy will be performed by observing either Schottky spectrum $[1,2]$ or recombination signal from debunched ions co-traveling with the e-beam. Yet, to start observing such signals one has to set absolute energy of electron beam with accuracy better than $10^{-2}$, preferably better than $5 \cdot 10^{-3}$.

The aim of this exercise is to determine whether and how such accuracy can be reached by utilizing LEReC $180^{\circ}$ bend as a spectrometer.

\section{0 degree bend}

The $180^{\circ}$ bend setup is schematically shown in Fig. 1 .

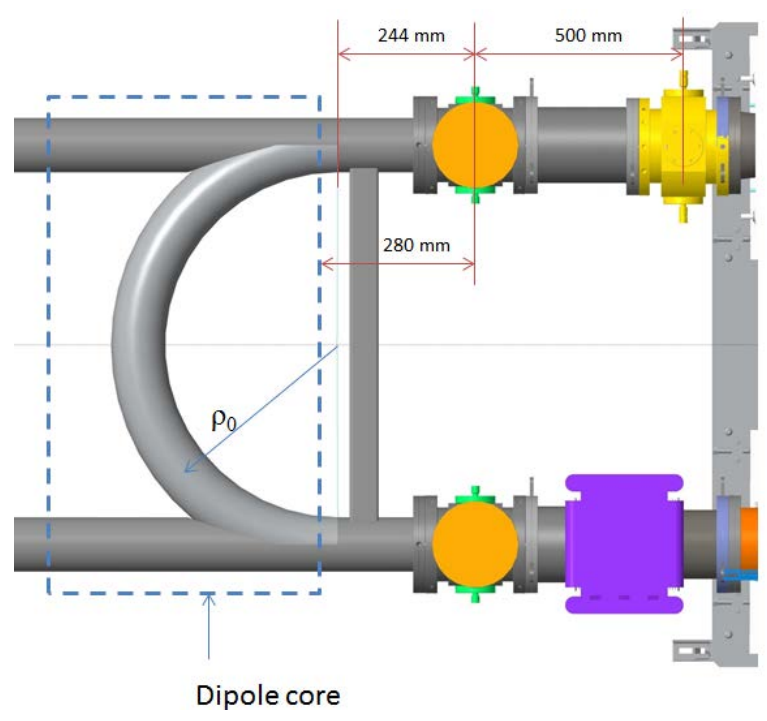

Figure 1: Schematics of $180^{\circ}$ bend. 
The $180^{\circ}$ bend is located between the first and the second LEReC cooling sections. It is designed to have a bending radius $\rho_{0}=0.35 \mathrm{~m}$. The entrance to the magnet is equipped with two BPMs (one of them hybrid [3]) and its exit is equipped with one hybrid BPM. BPM-to-BPM distances are defined by requirements to the precision of energy regulation [4] and were recently set in dedicated optical simulations [5].

\section{Hard-edge approximation}

It is not hard to see (Fig. 2) that in the hard-edge approximation the horizontal e-beam displacement $\left(x_{\text {out }}\right)$ at the exit of $180^{\circ}$ bend is given by:

$$
x_{\text {out }}=-x_{\text {in }}+2 \rho_{0}-2 \rho \cos \theta_{\text {in }}
$$

The notation used in (1) is explained in Fig. 2.

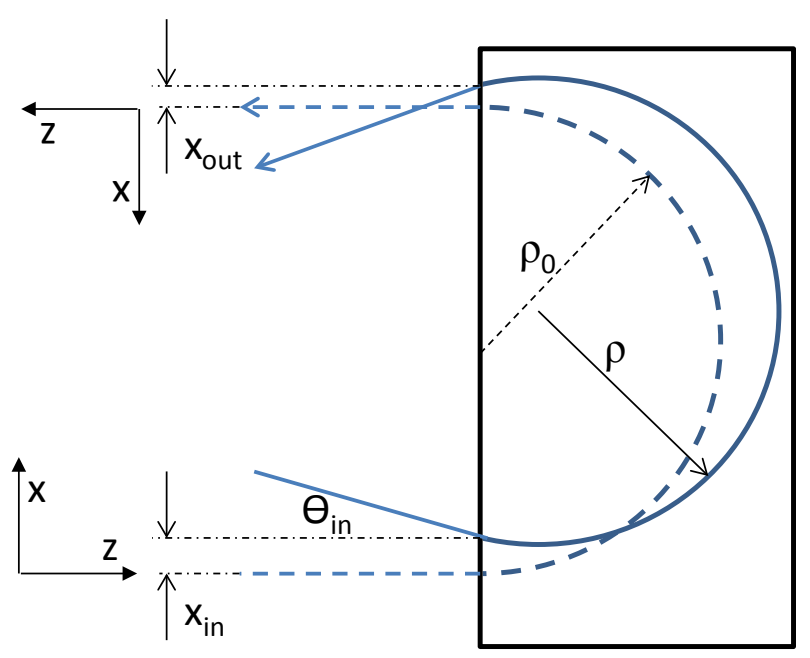

Figure 2: Beam trajectory in $180^{\circ}$ bend.

Cosine in (1) can be substituted by 1 unless $\theta_{\text {in }}$ is on the order of $0.01 \mathrm{rad}$.

Usually one would approximate magnetic rigidity as:

$$
\frac{B \rho}{B_{0} \rho_{0}} \approx \frac{E}{E_{0}} \equiv 1+\delta
$$

thus obtaining standard expression for dispersion $D=-2 \rho_{0} B_{0} / B$ (or $D=-2 \rho_{0}$ for nominal dipole field) and finding error in energy setting (for $\left.B=B_{0}\right)$ as $\delta=\left(x_{\text {out }}+x_{i n}\right) / D$. Yet, for our range of energies (1.6 MeV $-2.1 \mathrm{MeV}$ ) the resulting error in found energy would be too large as plot in Fig. 3 demonstrates. 


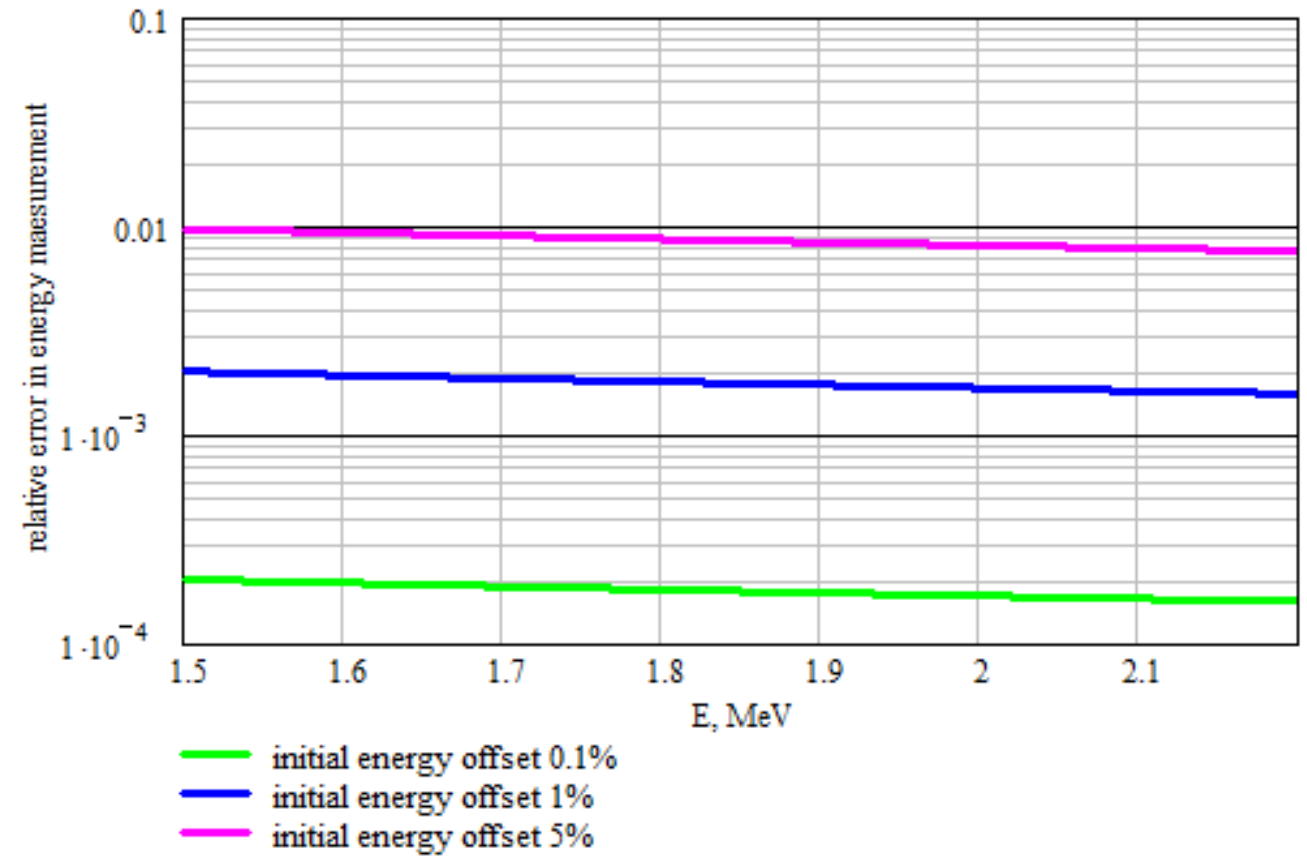

Figure 3: Relative error in measured energy (when utilizing standard simplified expression for D) for various initial offsets of the real beam energy.

To measure real beam energy with required accuracy of about $0.1 \%$ one, instead of using (2), must perform proper Taylor expansion of exact expression for magnetic rigidity:

$$
B \rho=\frac{m c}{e} \sqrt{\left(\frac{E_{0}(1+\delta)}{m c^{2}}+1\right)^{2}-1}
$$

Expanding (3) with small parameter $\delta$ we obtain:

$$
B \rho=B_{0} \rho_{0}\left(1+\frac{E_{0}+m c^{2}}{E_{0}+2 m c^{2}} \delta\right)
$$

Substituting (4) into (1) for $B=B_{0}$ we get:

$$
x_{\text {out }}=-x_{\text {in }}-2 \rho_{0} \frac{E_{0}+m c^{2}}{E_{0}+2 m c^{2}} \delta
$$

Utilizing (5) one can measure the real beam energy with better than $10^{-4}$ accuracy (under the worst case scenario) as the plot in Fig. 4 demonstrates. 


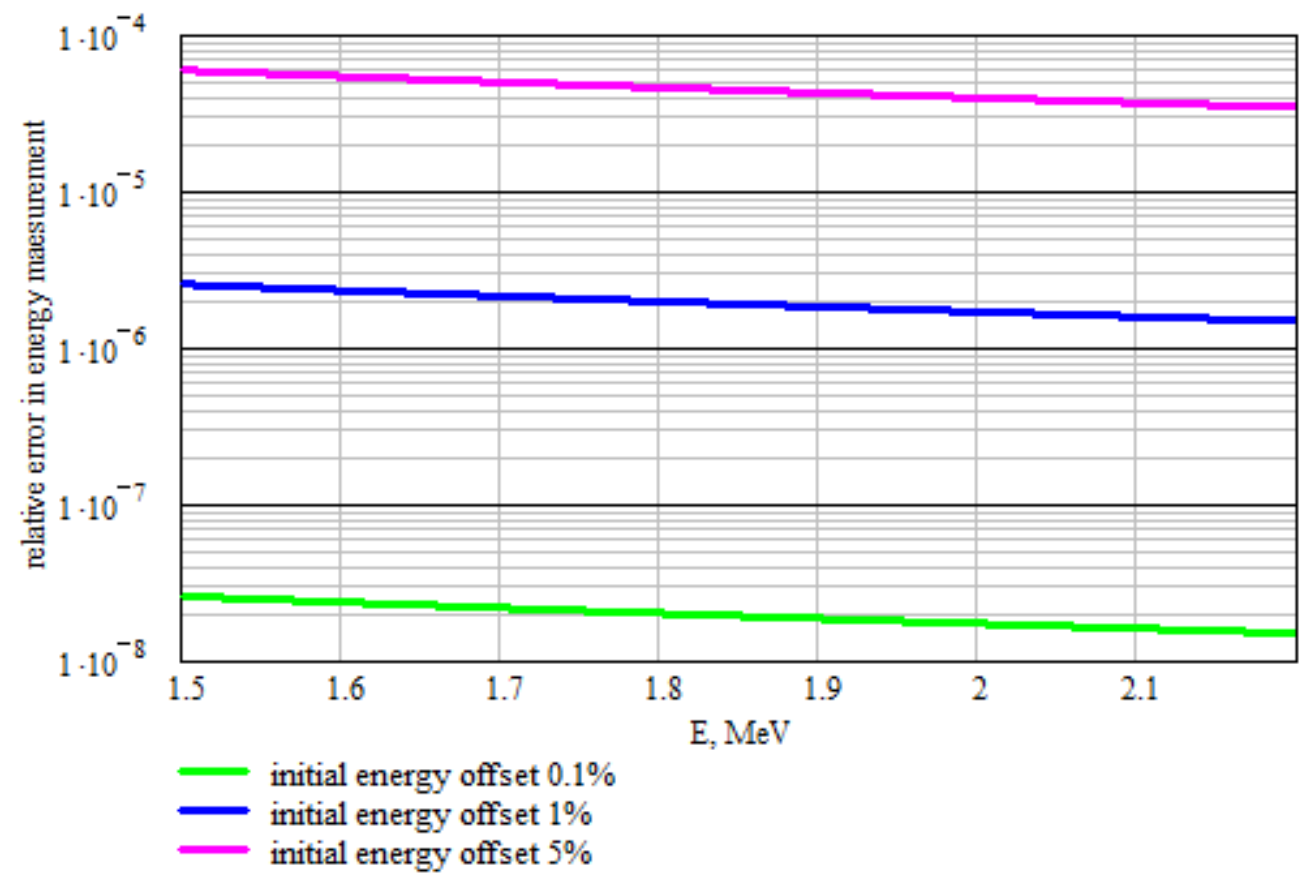

Figure 4: Relative error in measured energy (we are utilizing proper formula (5) to measure the energy) for various initial offsets of the real beam energy.

Finally, defining fractional error in setting dipole field as $\Delta \equiv B / B_{0}-1$, from (1) and (4) we get:

$$
x_{\text {out }}=-x_{\text {in }}-2 \rho_{0} \frac{E_{0}+m c^{2}}{E_{0}+2 m c^{2}} \delta+2 \rho_{0} \Delta
$$

Equation (6) solves the problem of the dependence of accuracy of energy measurement on accuracies of BPM settings and magnetic field measurement. Indeed, since $x_{\text {in }}$ and $x_{\text {out }}$ correspond to BPM readings $x_{1}, x_{2}, x_{3}$ (see Fig. 5 for details) as:

$$
\begin{aligned}
& x_{\text {in }}=x_{2}+S_{b} \frac{x_{2}-x_{1}}{S_{12}} \\
& x_{\text {out }}=x_{3}+S_{b} \frac{x_{2}-x_{1}}{S_{12}}
\end{aligned}
$$

the accuracy of energy measurement $\left(\tilde{\delta} \equiv\left(E_{\text {measured }}-E_{\text {real }}\right) / E_{0}\right)$ is given by:

$$
\tilde{\delta}=\frac{E_{0}+2 m c^{2}}{E_{0}+m c^{2}}\left(\Delta+\frac{\sigma_{B P M}}{\rho_{0}}\left(1+2 \frac{S_{b}}{S_{12}}\right)\right)
$$


Here $\sigma_{B P M}$ is the absolute error of BPM readings, which includes accuracy of BPM alignment with respect to the other and with respect to the dipole and the reading accuracy per se. Assuming 1.6 MeV nominal energy of the beam (the worst case) we get:

$$
\tilde{\delta}=1.24 \Delta+7.52 \sigma_{B P M}
$$

If the accuracy of magnetic field measurement/setting is $10^{-3}$ and $\sigma_{B P M}=0.1 \mathrm{~mm}$ then $\tilde{\delta}=2 \cdot 10^{-3}$. For the case of relaxed requirements of BPM measurement/setting error of $\sigma_{B P M}=0.5 \mathrm{~mm}$ we get $\tilde{\delta}=5 \cdot 10^{-3}$.

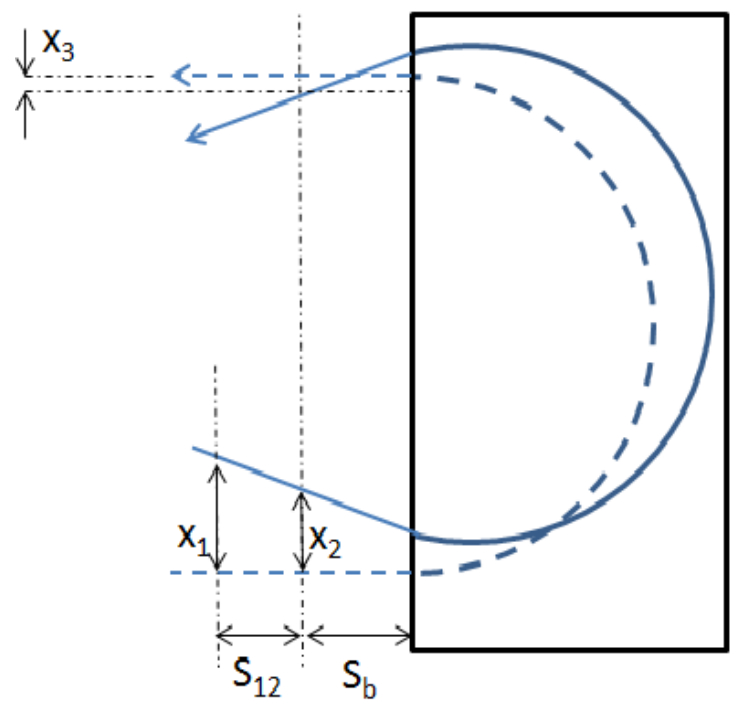

Figure 5: Schematics of BPM settings with respect to the dipole.

\section{Dipole with soft-edge field}

The real dipole has a soft-edge field. While formulas (6-10) might be a good approximation of beam dynamics in the real dipole it is worth to simulate beam trajectory in the soft-edge dipole, introduce the errors in BPM readings and field settings and see with what accuracy we can measure beam energy in real life.

The algorithm of beam dynamics simulation in varying dipole field is discussed in Appendix A. Here we will consider the results of these simulations for the case of $1.6 \mathrm{MeV}$ beam.

We choose such strength of realistic dipole field that the resulting dispersion downstream of the bend is equal to nominal $70 \mathrm{~cm}$. Figure 6 shows the field along beam trajectory and the actual beam trajectory between BPMs 2 and 3 along with the field and trajectory in equivalent hard-edge dipole.

Now, we will consider various measurement and setting errors, which affect the accuracy of energy measurement. 

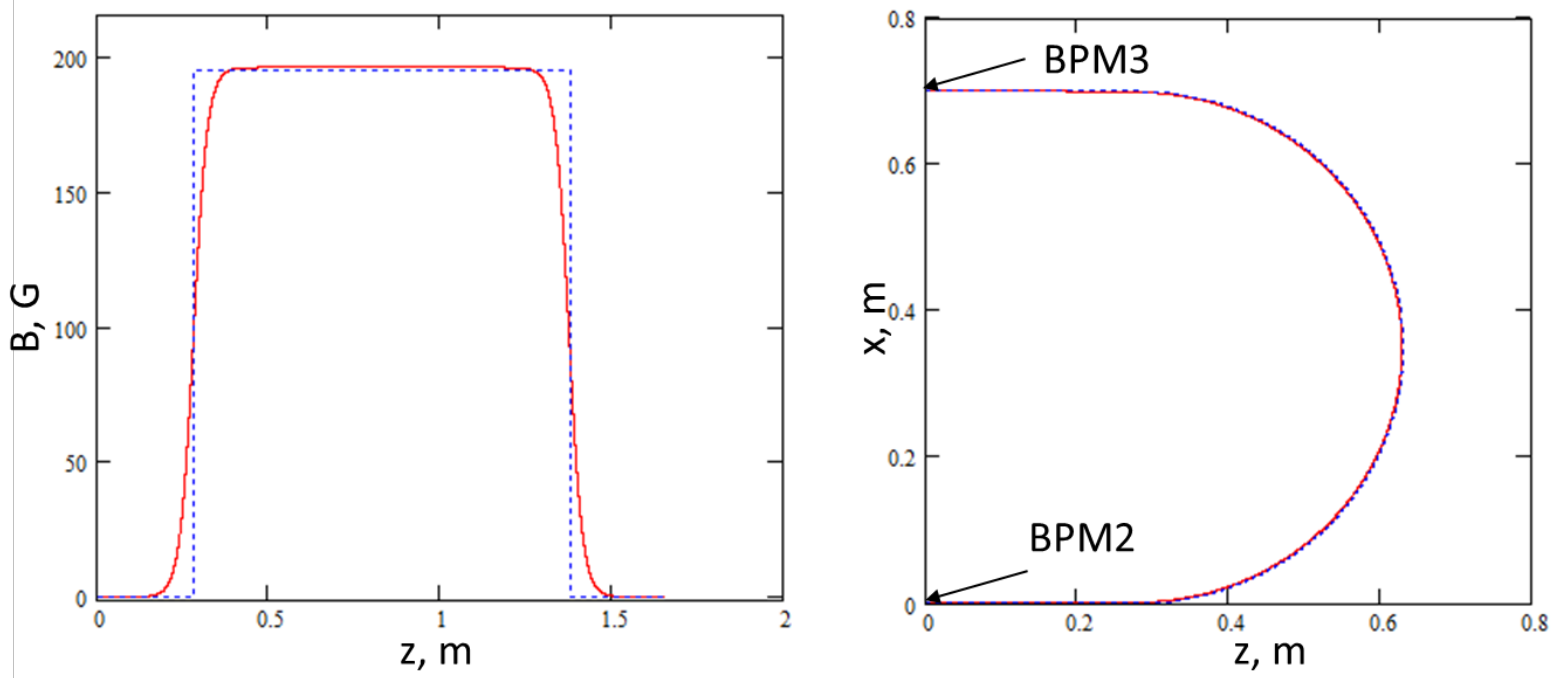

Figure 6: Dipole magnetic field along e-beam trajectory (left) and e-beam trajectory from BPM 2 to BPM 3 (right). The solid red line represents simulation results for the realistic soft-edge dipole. The dotted blue line represents simulation results for equivalent hard-edge approximation.

\section{1.) Accuracy of the magnetic probe}

The dipole field will be mapped in the region covering possible trajectories of the electron beam with magnetic probe - NMR probe in homogeneous field region and Hall probe in the edges. For NMR typical measurement accuracy is $10^{-4}$ or $0.02 \mathrm{G}$ for $\sim 200 \mathrm{G}$ filed. We believe that since the Hall probe is installed together with NMR in the same holder and can be calibrated vs. NMR in homogeneous field, the accuracy of edge field measurement also will be $0.02 \mathrm{G}$. We also consider the worst case scenario assuming $0.1 \mathrm{G}$ accuracy of the field measurement.

Therefore, in our simulations we introduce $0.02 \mathrm{G}$ (0.1 G for worst case scenario) of systematic "shift" in the realistic dipole field.

\section{2.) Inclination of magnetic probe with respect to the dipole field}

If the axis of magnetic probe is inclined with respect to the dipole field by angle $\alpha$ then the measured field is reduced by $\cos (\alpha)$. In simulations we will assume this angle to be $1^{\circ}$ ( $2^{\circ}$ for the worst case scenario).

\section{3.) Accuracy of setting dipole current}

We procured the dipole power supply, which provides $3 \cdot 10^{-5}$ accuracy. We also will develop and implement an automated hysteresis cycling system to set the dipole operating point. In simulations we assume overall accuracy of field setting to be $10^{-4}$.

\section{4.) Field quality (asymmetry of the edges)}

While ideal $180^{\circ}$ bend is a magnetic mirror, the real dipole field is not ideally symmetric. This is especially true for symmetry of the entrance/exit edge fields. 
In principal, if this asymmetry is properly measured, than one can account for it in simulations and adjust dipole current accordingly. In such scenario one still gets nominal dispersion $D$ in BPM3. Yet, there also is nonzero $D^{\prime}$ downstream of the dipole. If $D^{\prime}$ is small enough it will neither affect energy measurement, nor hinder the cooling process in the second CS.

For simulations we will assume that possible misbalance in edge fields (see Fig. 7 for details) is not higher than $0.5 \mathrm{G}$ (and we are not "compensating" resulting dispersion change).

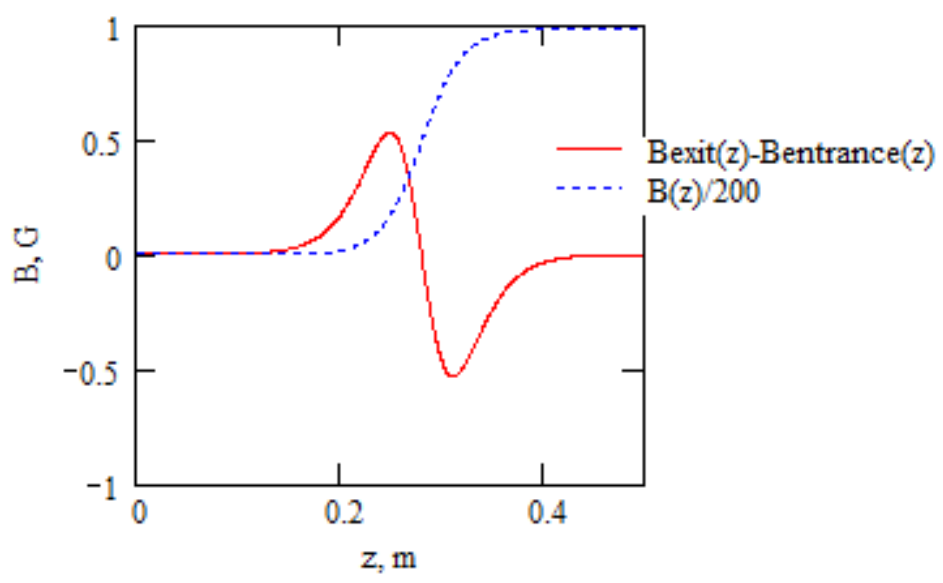

Figure 7: The difference between asymmetric entrance and exit edge fields (solid red line). For the reference the dotted blue line shows the edge field profile (divided by 200).

\section{5.) Ambient magnetic field}

There is an ambient magnetic field present in the RHIC tunnel. It is probable that the dipole will be measured in the magnetic lab only and that this measurement will not be repeated inside the tunnel. The ambient field shall be well shielded inside the dipole and not that well shielded around dipole entrance and exit.

The transverse field at dipole location inside RHIC tunnel was measured to be about $0.35 \mathrm{G}$. For our simulations we assume that the unaccounted for dipole field of $0.4 \mathrm{G}$ is present in $35 \mathrm{~cm}$ long regions downstream of BPM 2 and upstream of BPM 3.

\section{6.) Beam trajectory errors}

Finally, there are errors in BPM readings due to reading accuracy (cable length, electronics noise etc.) and due to the errors in BPMs positioning both with respect to each other and with respect to the mapped dipole field. In our simulations we assume that all these errors result in BPM readings accuracy to be $0.1 \mathrm{~mm}(0.5 \mathrm{~mm}$ in the worst case scenario).

We suggest that for the sake of simplicity of interpreting results energy measurement is done for zeroed entrance angle of the beam trajectory. Assuming that BPMs 1 and 2 are 
set with $0.1 \mathrm{~mm}$ accuracy we get the entrance angle with 0.4 mrad accuracy $(2 \mathrm{mrad}$ for the worst case scenario).

We introduce all the described errors to our simulations in such fashion that the total resulting measurement error is maximized. Next, we simulate $5 \%$-off-energy beam trajectory in the dipole (Fig. 8) and calculate the measured energy according to (5) with $x_{\text {in }}=x_{2}=0$ and $x_{\text {out }}=x_{3}$, assuming $0.1 \mathrm{~mm}$ accuracy of BPM readings.

As a result we measure the real beam energy with $2.6 \cdot 10^{-3}$ accuracy. In the worst case scenario the measurement accuracy becomes $6.7 \cdot 10^{-3}$.

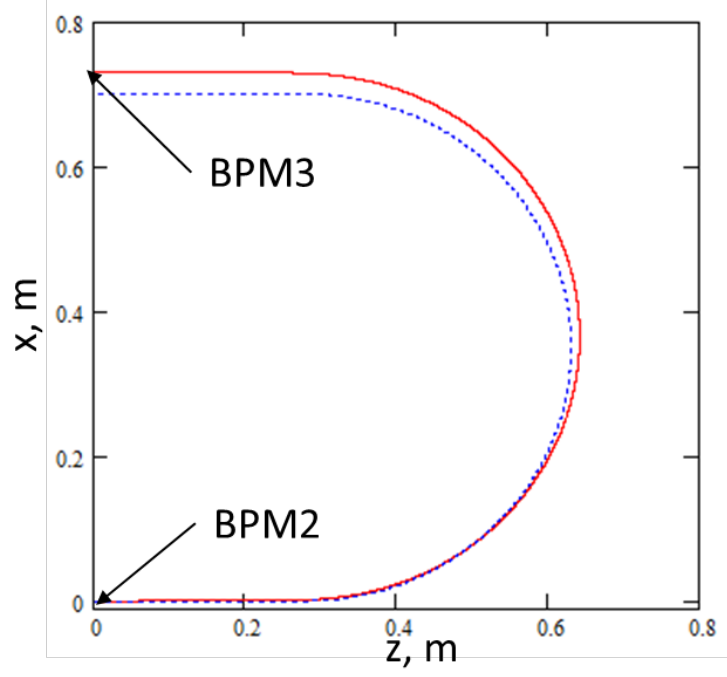

Figure 8: 5\% off energy beam trajectory (solid red line) and reference on-energy beam trajectory (dotted blue line) in realistic soft-edge dipole. The off-energy beam trajectory includes all errors listed above.

\section{Conclusion}

We estimated the accuracy of absolute energy measurement utilizing LEReC $180^{\circ}$ dipole magnet. We performed both the analytical estimates in hard-edge bend approximation and simulations of beam trajectory in realistic soft-edge dipole field.

We conclude that assuming various errors listed in Table 1 we expect measuring beam energy with $2.6 \cdot 10^{-3}$ accuracy. In the worst case scenario we can measure beam energy with $6.7 \cdot 10^{-3}$ accuracy.

It is essential to use formula (5) to calculate real beam energy. 


\begin{tabular}{|l|l|l|l|l|l|l|l|}
\hline $\begin{array}{l}\text { Magnetic } \\
\text { probe } \\
\text { accuracy }\end{array}$ & $\begin{array}{l}\text { Magnetic } \\
\text { probe } \\
\text { inclination }\end{array}$ & $\begin{array}{l}\text { Dipole } \\
\text { current } \\
\text { accuracy }\end{array}$ & $\begin{array}{l}\text { Edge field } \\
\text { asymmetry }\end{array}$ & $\begin{array}{l}\text { Ambient } \\
\text { field }\end{array}$ & $\begin{array}{l}\text { BPM } \\
\text { accuracy }\end{array}$ & $\begin{array}{l}\text { Energy } \\
\text { error, } \\
\text { simulation }\end{array}$ & $\begin{array}{l}\text { Energy } \\
\text { error, } \\
\text { analytic }\end{array}$ \\
\hline $0.02 \mathrm{G}$ & $1^{\circ}$ & $10^{-4}$ & $0.5 \mathrm{G}$ & $0.4 \mathrm{G}$ & $0.1 \mathrm{~mm}$ & $\mathbf{2 . 6 \cdot 1 0 ^ { - 3 }}$ & $\mathbf{2 \cdot 1 0 ^ { - 3 }}$ \\
\hline $0.1 \mathrm{G}$ & $2^{\circ}$ & $10^{-4}$ & $0.5 \mathrm{G}$ & $0.4 \mathrm{G}$ & $0.5 \mathrm{~mm}$ & $\mathbf{6 . 7 \cdot 1 0 ^ { - 3 }}$ & $\mathbf{5 \cdot 1 0 ^ { - 3 }}$ \\
\hline
\end{tabular}

Table 1: Upper limit on various errors affecting accuracy of beam energy measurement

\section{Acknowledgements}

The author is grateful for the input to this paper from the whole LEReC team. Special thanks go to Peter Thieberger and Alexei Fedotov.

\section{References}

[1] S. Nagaitsev et al., PRL 96, 044801 (2006).

[2] S. Seletskiy, Attainment of Electron Beam Suitable for Medium Energy Electron Cooling (see Chapter 5), PhD thesis, 2005.

[3] T. Miller et al., Multifunction instrument designs with low impedance structures for profile, energy, and emittance measurements for LEReC at BNL, Proceedings of IBIC15, 2015.

[4] P. Thieberger, LEReC energy regulation issues, 1/6/2016.

[5] D. Kayran, LEReC: U-turn magnet edge effect and its compensation, 03/28/2016. 


\section{Appendix A: Proper simulations of beam trajectory in dipole field}

Since we are interested in rather accurate measurement of beam energy it is essential to perform proper simulation of beam motion in the dipole field without neglecting "small" values acquired at each infinitesimal step. We suggest using the algorithm described by the formulas listed below and geometrically explained in Fig. A1.

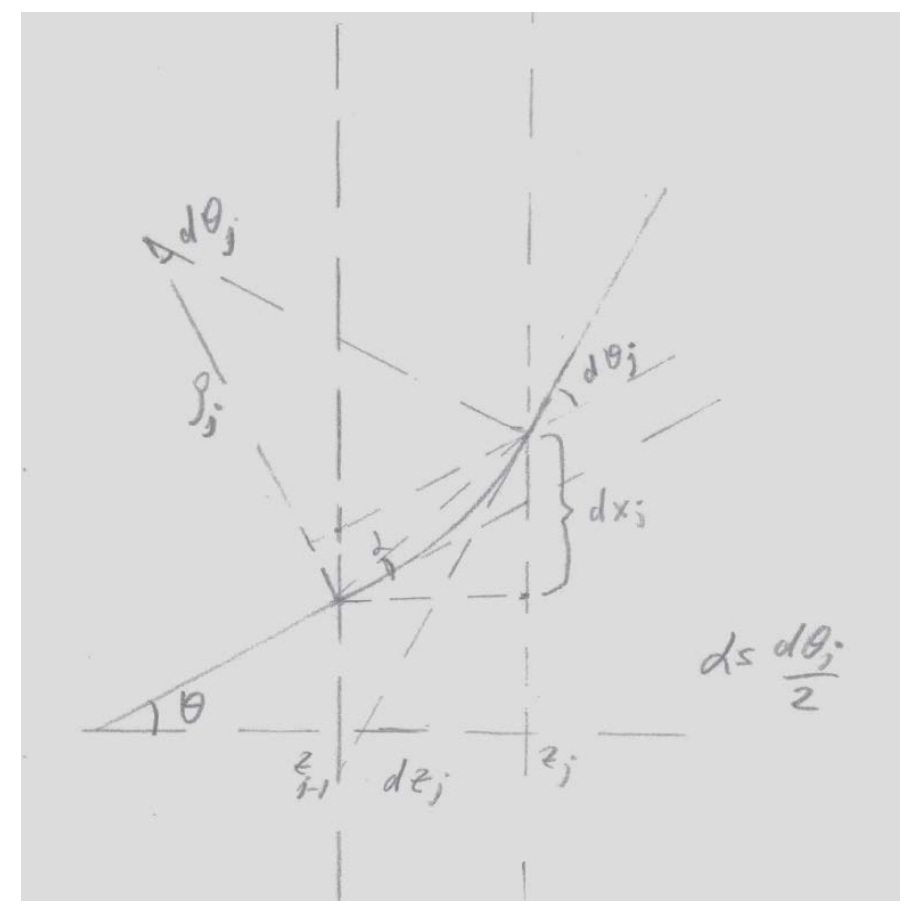

Figure 1A: Beam trajectory through one infinitesimal step $d s$.

In the following formulas $j$ is the step number. The notations are explained in Fig. 1A.

$$
\begin{gathered}
\rho_{j}=\frac{B \rho}{B\left(z_{j-1}\right)}, \quad d \theta_{j}=\frac{d s}{\rho_{j}} \\
d z_{j}=\rho_{j}\left[\sin \left(\theta_{j-1}+d \theta_{j}\right)-\sin \left(\theta_{j-1}\right)\right] \\
d x_{j}=\rho_{j}\left[\cos \left(\theta_{j-1}\right)-\cos \left(\theta_{j-1}+d \theta_{j}\right)\right] \\
z_{j}=z_{j-1}+d z_{j}, \quad x_{j}=x_{j-1}+d x_{j}, \quad \theta_{j}=\theta_{j-1}+d \theta_{j}
\end{gathered}
$$

The only requirement to this algorithm is that $d s$ is small enough that change in $B$ over one step is negligible. For our simulations we initially choose $d s=1 \mu \mathrm{m}$. For such choice the relative change in magnetic field never exceeds $5 \cdot 10^{-5}$. Eventually, we increased $d s$ to $10 \mu \mathrm{m}$, checking that the results of simulations do not change. 


\section{Appendix B: Algorithm of energy measurement}

Below we suggest an algorithm for absolute measurement of beam energy.

\section{Prerequisites:}

1.) Dipole field is mapped with $0.02 \mathrm{G}$ accuracy.

2.) The hysteresis cycling for precise setting of dipole working point has being worked out.

3.) Two BPMs in front of the dipole (BPMs 1\&2) and one BPM downstream of the dipole (BPM 3) are set with the accuracy of $0.1 \mathrm{~mm}$ with respect to one another and with respect to the mapped dipole field.

\section{Measurement procedure:}

1.) Simulate beam motion in the measured dipole field. For the nominal beam energy set dipole current so that the dispersion at BPM 3 location is equal to the nominal $70 \mathrm{~cm}$ dispersion.

2.) Utilizing CS correctors zero both the horizontal beam displacement in BPM 2 and beam trajectory angle in BPM1-BPM2 drift.

3.) Measure obtained beam displacement in BPM3.

4.) Apply equation (5) to calculate beam energy.

As a final note, we might want to change the current design of BPM 1 from regular BPM to hybrid BPM. 